\title{
THE GENERAL PROBLEM OF LONG WAVES ON A ROTATING EARTH*
}

\author{
BY \\ LL. G. CHAMBERS \\ University College of North Wales, Bangor, Wales
}

Summary. A method is given which makes it possible to calculate the surface elevation associated with long waves on a rotating earth when fluid is created or passes over geometrical boundaries.

1. Introduction. The equations associated with the classical long wave theory have been given by Proudman [1], and have been extended by Chambers [2] to include the case where the continuity equation does not hold.

It is assumed that $\mathbf{q}$, the horizontal fluid velocity, is independent of depth; that $h$, the sea depth, is constant; and that the fluid is uniform. It is further assumed that variations in the Coriolis parameter are negligible and that the surface of the earth is sensibly plane.

Under these conditions, the horizontal equation of motion is

$$
\partial q / \partial t+\Omega \mathbf{k} \times \mathbf{q}=-g \nabla \zeta
$$

where $\zeta$ is the elevation of the free surface above its mean level, $\nabla$ the two-dimensional gradient operator, $\mathbf{k}$ the vertically upward unit vector and $\Omega$ the Coriolis parameter ( $\Omega=2 \omega_{0} \sin \alpha$ where $\omega_{0}$ is the angular velocity of the earth and $\alpha$ the north latitude).

If a volume $V_{0}$ of fluid is liberated uniformly over the depth of the fluid at zero time along the $z$ axis, the solution of Eq. (1) is given through a potential $A$ such that

$$
\mathbf{q}=\frac{\partial}{\partial t}(\nabla A)-\Omega(\mathbf{k} \times \nabla A)
$$

where $A$ is defined by

$$
\nabla^{2} A-K^{2} A-\frac{1}{c^{2}} \frac{\partial^{2} A}{\partial t^{2}}=\frac{V_{0}}{h} \delta(\mathbf{r}) H(t)
$$

where $c^{2}=g h, K^{2}=\Omega^{2} / c^{2}, \delta(\mathbf{r})$ is the two-dimensional delta function and $H(t)$ is the Heaviside step function.

Frcm now on, the assumption will be made that all quantities are independent of $z$.

Suppose now that a volume of fluid $h \chi\left(\mathbf{r}^{\prime}, t^{\prime}\right) \delta x^{\prime} \delta y^{\prime} \delta t^{\prime}$ is liberated within the cylinder $x^{\prime}<x<x^{\prime}+\delta x^{\prime}, y^{\prime}<y<y^{\prime}+\delta y^{\prime}$ between times $t^{\prime}$ and $t^{\prime}+\delta t^{\prime}$.

* Received July 7, 1968; revised version received April 19, 1969. 
By Eq. (3), the associated potential $\delta A$ is given by

$$
\left(\nabla^{2}-K^{2}-\frac{1}{c^{2}} \frac{\partial^{2}}{\partial t^{2}}\right) \delta A=\chi\left(r^{\prime}, t^{\prime}\right) \delta x^{\prime} \delta y^{\prime} \delta t^{\prime} \delta\left(\mathbf{r}-\mathbf{r}^{\prime}\right) H\left(t-t^{\prime}\right),
$$

whence

$$
\begin{aligned}
\left(\nabla^{2}-K^{2}-\frac{1}{c^{2}} \frac{\partial^{2}}{\partial t^{2}}\right) A & =\int_{-\infty}^{\infty} \chi\left(\mathbf{r}, t^{\prime}\right) H\left(t-t^{\prime}\right) d t^{\prime} \\
& =\psi(\mathbf{r}, t), \text { say. }
\end{aligned}
$$

If $\chi$ vanishes for negative time, so also does $\psi$. It will be assumed that this is the case. The elevation $\zeta$ of the free surface above its mean level is given by

$$
\zeta=-h\left[K^{2} A+\frac{1}{c^{2}} \frac{\partial^{2} A}{\partial t^{2}}\right] .
$$

If the volume flow of fluid in across an element $\delta s$ of a geometrical boundary is given by $\theta h \delta s, \theta$ is the inward normal component of velocity $(-\mathbf{q} \cdot \mathbf{n})$ at the boundary, and so it follows from Eq. (2) that

$$
\frac{\partial^{2} A}{\partial n \partial t}+\Omega \frac{\partial A}{d s}=-\theta
$$

over a geometrical boundary. Where the geometrical boundary coincides with a barrier, $\theta$ is zero.

The determination of $A$ from the differential equation (4) and the boundary condition (5), together with initial conditions

$$
\begin{aligned}
A(\mathbf{r}, 0) & =A_{0}(\mathbf{r}) \\
{\left[\frac{\partial}{\partial t} A(\mathbf{r}, t)\right]_{t=0} } & =A_{1}(\mathbf{r}),
\end{aligned}
$$

may be referred to as the general problem of long waves on a rotating earth.

$\psi$ may represent movements of the sea bed and $\theta$ will correspond to flows across geometrical boundaries.

2. Integration of equation. In order to integrate the differential equation (4) it is necessary to use the Green's function $G\left(r, r^{\prime} ; t\right)$ which obeys the differential equation

$$
\left(\nabla^{2}-K^{2}-\frac{1}{c^{2}} \frac{\partial^{2}}{\partial t^{2}}\right) G=\delta\left(\mathbf{r}-\mathbf{r}^{\prime}\right) \delta(t),
$$

the conjugate boundary condition

$$
\frac{\partial^{2} G}{\partial n \partial t}-\Omega \frac{\partial G}{\partial s}=0
$$

and the initial conditions

$$
G=0, \quad \frac{\partial G}{\partial t}=0 \quad \text { at } \quad t=0 .
$$

The integration proceeds by the method of Ainola [3]. Define the convolution of two functions of time $f(t), g(t)$ in the usual way: 


$$
f * g=\int_{0}^{t} f\left(t-t^{\prime}\right) g\left(t^{\prime}\right) d t^{\prime}
$$

It is well known that the convolution obeys the commutative and associative laws.

Three particular cases are

$$
\begin{aligned}
1 * g & =\int_{0}^{t} g\left(t^{\prime}\right) d t^{\prime} . \\
\delta * g & =\int_{0}^{t} \delta\left(t-t^{\prime}\right) g\left(t^{\prime}\right) d t^{\prime} \\
& =g(t) . \\
1 * \frac{\partial f}{\partial t} & =\int_{0}^{t} \frac{\partial f}{\partial t} d t=f(t)-f(0) .
\end{aligned}
$$

$f$ and $g$ may, of course, be functions of other variables.

It can be shown, in extension of Green's theorem in two dimensions, that

$$
\begin{aligned}
\int\left[G * 1 * 1 * \nabla^{2} A-A * 1 * 1 * \nabla^{2} G\right] d x d y \\
=\int\left(G * 1 * 1 * \frac{\partial A}{\partial n}-A * 1 * 1 * \frac{\partial G}{\partial n}\right) d s
\end{aligned}
$$

Now

$$
\begin{aligned}
& \nabla^{2} A=K^{2} A+\frac{1}{c^{2}} \frac{\partial^{2} A}{\partial t^{2}}+\psi \\
& \nabla^{2} G=K^{2} G+\frac{1}{c^{2}} \frac{\partial^{2} G}{\partial t^{2}}+\delta\left(\mathbf{r}-\mathbf{r}^{\prime}\right) \delta(t)
\end{aligned}
$$

The left-hand side of (12) becomes

$$
\begin{aligned}
\int\left\{G * 1 * 1 * \left[K^{2} A+\right.\right. & \left.\frac{1}{c^{2}} \frac{\partial^{2} A}{\partial t^{2}}+\psi\right] \\
& \left.-A * 1 * 1 *\left[K^{2} G+\frac{1}{c^{2}} \frac{\partial^{2} G}{\partial t^{2}}+\delta\left(\mathbf{r}-\mathbf{r}^{\prime}\right) \delta(t)\right]\right\} d x d y \\
= & \frac{1}{c^{2}} \int\left(G * 1 * 1 * \partial^{2} A / \partial t^{2}-A * 1 * 1 * \partial^{2} G / \partial t^{2}\right) d x d y \\
& +\int G * 1 * 1 * \psi d x d y \\
& -A\left(r^{\prime}, t\right) * 1 * 1 * \delta(t)
\end{aligned}
$$

using the relation $\int f(\mathbf{r}) \delta\left(\mathbf{r}-\mathbf{r}^{\prime}\right) d x d y=f\left(\mathbf{r}^{\prime}\right)$. Now

$$
\begin{aligned}
1 * 1 * \partial^{2} A / \partial t^{2} & =1 *\left[\partial A / \partial t-A_{1}(\mathrm{r})\right] \\
& =A-A_{0}(\mathrm{r})-t A_{1}(\mathrm{r}) .
\end{aligned}
$$


Similarly $1 * 1 *\left(\partial^{2} G / \partial t^{2}\right)=G$ using the initial conditions. Also

$$
\begin{aligned}
A\left(\mathbf{r}^{\prime}, t\right) * 1 * 1 * \delta(t) & =1 * 1 *\left[A\left(\mathbf{r}^{\prime}, t\right) * \delta(t)\right] \\
& =1 * 1 * A\left(\mathbf{r}^{\prime}, t\right)
\end{aligned}
$$

and so the left-hand side of (12) becomes

$$
\int G *\left[1 * 1 * \psi-\frac{1}{c^{2}}\left\{t A_{1}(\mathrm{r})-A_{0}(\mathrm{r})\right\}\right] d x d y-1 * 1 * A\left(r^{\prime}, t\right)
$$

the other terms cancelling out.

The right-hand side of (12) may be rewritten

$$
\int 1 * 1 *\left[G * \frac{\partial A}{\partial n}-A * \frac{\partial G}{\partial n}\right] d s
$$

Now

$$
1 * \frac{\partial^{2} A}{\partial n \partial t}=\frac{\partial A}{\partial n}-\left[\frac{\partial A}{\partial n}\right]_{t=0}
$$

and

$$
1 * \frac{\partial^{2} G}{\partial n \partial t}=\frac{\partial G}{\partial n}-\left[\frac{\partial G}{\partial n}\right]_{t=0} .
$$

Now at $t=0, A$ is known everywhere and so therefore $\partial A / \partial n$ is known. Similarly $G$ is then zero everywhere and so $\partial G / \partial n$ is zero. Thus, the right-hand side of (12) becomes

$$
\begin{aligned}
\int 1 * 1 *\left[G *\left\{1 * \frac{\partial^{2} A}{\partial n \partial t}+\left(\frac{\partial A}{\partial n}\right)_{t=0}\right\}-\right. & \left.A * 1 * \frac{\partial^{2} G}{\partial n \partial t}\right] \\
= & -\int 1 * 1 * 1 *\left\{G * \frac{\partial A}{\partial s}+A * \frac{\partial G}{\partial s}\right\} \Omega d s \\
& +\int 1 * 1 * G *\left(\frac{\partial A}{\partial n}\right)_{t=0} d s \\
& -\int 1 * 1 * G * 1 * \theta d s,
\end{aligned}
$$

using Eqs. (5) and (8). Now

$$
G * \frac{\partial A}{\partial s}+A * \frac{\partial G}{\partial s}=\frac{\partial}{\partial s}(G * A)
$$

and so the first integral vanishes on integration round the boundary. Thus the right-hand side of (12) assumes the form

$$
-\int 1 * 1 * G *\left(1 * \theta+\left[\frac{\partial A}{\partial n}\right]_{l=0}\right) d s .
$$

On equating the expressions (13) and (14)

$$
\begin{aligned}
\int G *\left[1 * 1 * \psi-\frac{1}{c^{2}}\left\{t A_{1}(\mathrm{r})-A_{0}(\mathrm{r})\right\}\right] d x d y-1 * 1 * A\left(\mathbf{r}^{\prime}, t\right) & \\
= & -\int 1 * 1 * G *\left\{1 * \theta+\left(\frac{\partial A}{\partial n}\right)_{t=0}\right\} d s
\end{aligned}
$$


This may be rewritten in the following form:

$$
\begin{array}{rl}
1 * 1 * A\left(\mathbf{r}^{\prime}, t\right)=1 * 1 * \int G * \psi d & d y-\frac{1}{c^{2}} \int G *\left[t A_{1}(\mathbf{r})+A_{0}(\mathbf{r})\right] d x d y \\
+1 * 1 * \int G *\left\{1 * \theta+\left[\frac{\partial A}{\partial n}\right]_{t-0}\right\} d s
\end{array}
$$

From Eq. (11a)

$$
\frac{\partial}{\partial t}(1 * g)=g
$$

and differentiating Eq. (16) twice with respect to $t$, it follows that

$$
\begin{aligned}
A\left(\mathbf{r}^{\prime}, t\right)= & \int G * \psi d x d y \\
& +\int G *\left\{1 * \theta+\left(\frac{\partial A}{\partial n}\right)_{t=0}\right\} d s \\
& -\frac{1}{c^{2}} \frac{\partial^{2}}{\partial t^{2}} \int G *\left[t A_{1}(\mathbf{r})+A_{0}(\mathbf{r})\right] d x d y,
\end{aligned}
$$

thereby determining $A$ completely, and, through Eq. (5), $\zeta$. If now it be assumed that all the fluid is undisturbed prior to $t=0$, Eq. (17a) simplifies to

$$
A\left(\mathrm{r}^{\prime}, t\right)=\int G * \psi d x d y+\int G * 1 * \theta d s
$$

3. Green's function for an infinitely long canal. Consider a canal defined by the region $0<x<a$. The flow of another canal or of a river into it may be regarded as a flow across one of the geometrical boundaries $x=0$ or $x=a$. This will be amenable to the method outlined above and it is necessary to determine the appropriate Green's function. This is given by the relations for $G\left(x, y ; x^{\prime}, y^{\prime} ; t\right)$ :

$$
\begin{gathered}
\frac{\partial^{2} G}{\partial x^{2}}+\frac{\partial^{2} G}{\partial y^{2}}-K^{2} G-\frac{1}{c^{2}} \frac{\partial^{2} G}{\partial t^{2}}=\delta\left(x-x^{\prime}\right) \delta\left(y-y^{\prime}\right) \delta(t) \text { in } 0<x<a \\
\frac{\partial^{2} G}{\partial x \partial t}-\Omega \frac{\partial G}{\partial y}=0 \quad \text { on } \quad x=0, \quad x=a,
\end{gathered}
$$

and

$$
G=0, \quad \frac{\partial G}{\partial t}=0, \quad \text { at } \quad t=0 .
$$

Clearly $y^{\prime}$ may be assumed zero.

Applying the Laplace transform, let

$$
\bar{G}=\int_{0}^{\infty} e^{-p t} G(t) d t .
$$

(Quantities involving $p$ will be barred.) Then

$$
\begin{gathered}
\frac{\partial^{2} \bar{G}}{\partial x^{2}}+\frac{\partial^{2} \bar{G}}{\partial y^{2}}-\left(K^{2}+\frac{p^{2}}{c^{2}}\right) \bar{G}=\delta\left(x-x^{\prime}\right) \delta(y) \quad \text { in } 0<x<a . \\
p \frac{\partial \bar{G}}{\partial x}-\Omega \frac{\partial \bar{G}}{\partial y}=0 \quad \text { on } \quad x=0, \quad x=a .
\end{gathered}
$$


Let

$$
\bar{\Gamma}=p \frac{\partial \bar{G}}{\partial x}-\Omega \frac{\partial \bar{G}}{\partial y} .
$$

Then

$\frac{\partial^{2} \bar{\Gamma}}{\partial x^{2}}+\frac{\partial^{2} \bar{\Gamma}}{\partial y^{2}}-\left(K^{2}+\frac{p^{2}}{c^{2}}\right) \bar{\Gamma}=p \delta^{\prime}\left(x-x^{\prime}\right) \delta(y)-\Omega \delta\left(x-x^{\prime}\right) \delta^{\prime}(y) \quad 0<x<a$.

This is subject to the boundary condition

$$
\bar{\Gamma}=0 \quad \text { on } \quad x=0, \quad x=a .
$$

Thus if $G_{0}$ is the Green's function obeying the following boundary conditions:

$$
\begin{gathered}
\frac{\partial^{2} G_{0}}{\partial x^{2}}+\frac{\partial^{2} G_{0}}{\partial y^{2}}-\gamma^{2} G_{0}=\delta\left(x-x^{\prime}\right) \delta(y) \quad 0<x, \quad x^{\prime}<a \\
G_{0}=0 \text { on } x=0, \quad x=a, \\
\gamma^{2}=K^{2}+p^{2} / c^{2}
\end{gathered}
$$

a solution of Eq. (21a) is

$$
\bar{\Gamma}=-\left(p \partial / \partial x^{\prime}+\Omega \partial / \partial y\right) G_{0} .
$$

By the usual Green's function techniques,

$$
G_{0}=-\frac{2}{a} \sum_{n=1}^{\infty} \frac{1}{\gamma_{n}} e^{-\gamma_{n}|\nu|} \sin \frac{n \pi x}{a} \sin \frac{n \pi x^{\prime}}{a}
$$

where

$$
\begin{aligned}
\gamma_{n}^{2} & =\gamma^{2}+\frac{n^{2} \pi^{2}}{a^{2}}=K^{2}+\frac{p^{2}}{c^{2}}+\frac{n^{2} \pi^{2}}{a^{2}} \\
& =-\sum_{n=1}^{\infty} \sin \frac{n \pi x}{a} \Phi_{n}(y)
\end{aligned}
$$

where

$$
\bar{\phi}_{n}(y)=\frac{2}{a \gamma_{n}} e^{-\gamma_{n}|\nu|} \sin \frac{n \pi x^{\prime}}{a} .
$$

The reason why $\bar{\Gamma}$ is taken as $-\left(p \partial / \partial x^{\prime}+\Omega \partial / \partial y\right) G_{0}$ instead of $(p \partial / \partial x-\Omega \partial / \partial y) G_{0}$ is that for this latter case the boundary condition for $\bar{\Gamma}$ on $x=0, x=a$ would not be fulfilled. Let

$$
\bar{G}=\sum_{n=1}^{\infty}\left(\bar{A}_{n} \cos \frac{n \pi x}{a}+\bar{B}_{n} \sin \frac{n \pi x}{a}\right) .
$$

Then

$$
\begin{aligned}
\bar{\Gamma} & =p \frac{\partial \bar{G}}{\partial x}-\Omega \frac{\partial \bar{G}}{\partial y} \\
& =\sum_{n=1}^{\infty}\left\{-\Omega \frac{\partial \bar{A}_{n}}{\partial y}+p \frac{n \pi}{a} \bar{B}_{n}\right\} \cos \frac{n \pi x}{a} \\
& +\sum_{n=1}^{\infty}\left\{-p \frac{n \pi}{a} \bar{A}_{n}-\Omega \frac{\partial \bar{B}_{n}}{\partial y}\right\} \sin \frac{n \pi x}{a}
\end{aligned}
$$


Thus, comparing Eq. (25b) with the value of $\bar{\Gamma}$ obtained by substituting relation (24b) into Eq. (23), it follows that

$$
\begin{gathered}
-\Omega \frac{\partial \bar{A}_{n}}{\partial y}+p \frac{n \pi}{a} \bar{B}_{n}=0 \\
-p \frac{n \pi}{a} \bar{A}_{n}-\Omega \frac{\partial \bar{B}_{n}}{\partial y}=\left(p \frac{\partial}{\partial x^{\prime}}+\Omega \frac{\partial}{\partial y}\right) \bar{\phi}_{n} .
\end{gathered}
$$

Let

$$
\begin{gathered}
\bar{A}_{n}=\left(p \frac{\partial}{\partial x^{\prime}}+\Omega \frac{\partial}{\partial y}\right) \bar{C}_{n} \\
\bar{B}_{n}=\left(p \frac{\partial}{\partial x^{\prime}}+\Omega \frac{\partial}{\partial y}\right) \bar{D}_{n} .
\end{gathered}
$$

Then

$$
\begin{gathered}
-\Omega \frac{\partial \bar{C}_{n}}{\partial y}+p \frac{n \pi}{a} \bar{D}_{n}=0 \\
-p \frac{n \pi}{a} \bar{C}_{n}-\Omega \frac{\partial \bar{D}_{n}}{\partial y}=\Phi_{n} .
\end{gathered}
$$

Now

$$
\bar{D}_{n}=\frac{\Omega a}{p n \pi} \frac{\partial \bar{C}_{n}}{\partial y}
$$

from Eq. (28a), and so Eq. (28b) becomes

$$
-\frac{p n \pi}{a} \bar{C}_{n}-\frac{\Omega^{2} a}{p n \pi} \frac{\partial^{2} \bar{C}_{n}}{\partial y^{2}}=\bar{\phi}_{n}
$$

or

$$
\frac{\partial^{2} \bar{C}_{n}}{\partial y^{2}}+\frac{p^{2} n^{2} \pi^{2}}{a^{2} \Omega^{2}} \bar{C}_{n}=-\frac{p n \pi}{\Omega^{2} a} \bar{\phi}_{n} .
$$

Now it may easily be shown that if

$$
\begin{gathered}
\frac{d^{2} u}{d \zeta^{2}}+\alpha^{2} u=e^{-\beta|\zeta|}, \\
u=\frac{1}{\alpha^{2}+\beta^{2}} e^{-\beta|\zeta|}+\frac{\beta}{\alpha\left(\alpha^{2}+\beta^{2}\right)} \sin \alpha|\zeta| .
\end{gathered}
$$

The complementary function is assumed to vanish, as it would represent a wave proceeding all the way along the canal and thus would not be caused by a source in the finite part of the canal. It follows that

$$
\left.\bar{C}_{n}=-\frac{p n \pi}{\Omega^{2} a}\left\{\frac{1}{p^{2} n^{2} \pi^{2} / a^{2} \Omega^{2}+\gamma_{n}^{2}} e^{-\gamma_{n}|\nu|}+\frac{\gamma_{n} \sin \frac{p n \pi}{\Omega a}|y|}{\frac{p n \pi}{a \Omega}\left(\frac{p^{2} n^{2} \pi^{2}}{a^{2} \Omega^{2}}+\gamma_{n}^{2}\right.}\right\}\right) \frac{2}{a \gamma_{n}} \sin \frac{n \pi x^{\prime}}{a},
$$


and using Eq. (28c)

$$
\left.\bar{D}_{n}=-\frac{1}{\Omega} \frac{\partial}{\partial y}\left\{\frac{1}{\frac{p^{2} n^{2} \pi^{2}}{a^{2} \Omega^{2}}+\gamma_{n}^{2}} e^{-\gamma_{n}|v|}+\frac{\gamma_{n} \sin \frac{p n \pi}{\Omega a}|y|}{\frac{p n \pi}{a \Omega}\left(\frac{p^{2} n^{2} \pi^{2}}{a^{2} \Omega^{2}}+\gamma_{n}^{2}\right.}\right)\right\} \frac{2}{a \gamma_{n}} \sin \frac{n \pi x^{\prime}}{a} .
$$

Let

$$
K^{2}+\frac{n^{2} \pi^{2}}{a^{2}}=\alpha_{n}^{2}, \quad \frac{n^{2} \pi^{2}}{a^{2} \Omega^{2}}+\frac{1}{c^{2}}=\beta_{n}^{2}
$$

then

$$
\gamma_{n}^{2}=p^{2} / c^{2}+\alpha_{n}^{2} .
$$

Then

$$
\begin{aligned}
& \bar{C}_{n}=-\frac{p n \pi}{\Omega^{2} a}\left\{\frac{e^{-\gamma n|\nu|}}{p^{2} \beta_{n}^{2}+\alpha_{n}^{2}}+\frac{\gamma_{n} \sin \frac{p n \pi}{\Omega a}|y|}{\underline{p} \frac{n \pi}{\Omega a}\left(p^{2} \beta_{n}^{2}+\alpha_{n}^{2}\right)}\right\} \frac{2}{a \gamma_{n}} \sin \frac{n \pi x^{\prime}}{a} \\
& \bar{D}_{n}=-\frac{1}{\Omega} \frac{\partial}{\partial y}\left\{\frac{e^{-\gamma_{n}|y|}}{p^{\frac{2}{2} \beta_{n}^{2}+\alpha_{n}^{2}}}+\frac{\gamma_{n} \sin \frac{p n \pi}{\Omega a}|y|}{\frac{p n \pi}{\Omega a}\left(p^{2} \beta_{n}^{2}+\alpha_{n}^{2}\right)}\right\} \frac{2}{a \gamma_{n}} \sin \frac{n \pi x^{\prime}}{a} .
\end{aligned}
$$

It follows from Eqs. (25a), (27a), (27b), (32a) and (32b) that

$$
\begin{aligned}
\bar{G}= & \left(p \frac{\partial}{\partial x^{\prime}}+\Omega \frac{\partial}{\partial y}\right) \\
& \left.\cdot \sum_{n=1}^{\infty} \cos \frac{n \pi x}{a}\left\{\frac{p n \pi}{\Omega^{2}} \frac{e^{-\gamma n|y|}}{p^{\frac{2}{2} \beta_{n}^{2}}+\alpha_{n}^{2}}+\frac{\gamma_{n} \sin \frac{p n \pi}{\Omega a}|y|}{\frac{p n \pi}{\Omega a}\left(p^{2} \beta^{2}+\alpha_{n}^{2}\right)}\right]\right\} \frac{2}{a \gamma_{n}} \sin \frac{n \pi x^{\prime}}{a} \\
& +\sin \frac{n \pi x}{a}\left\{\frac{1}{\Omega} \frac{\partial}{\partial y}\left[\frac{e^{-\gamma_{n}|v|}}{p^{2} \beta_{n}^{2}+\alpha_{n}^{2}}+\frac{\gamma_{n} \sin \frac{p n \pi}{\Omega a}|y|}{\frac{p n \pi}{a}\left(p^{2} \beta^{2}+\alpha_{n}^{2}\right)}\right]\right\}\left(\frac{2}{a \gamma_{n}} \sin \frac{n \pi x^{\prime}}{a} .\right.
\end{aligned}
$$

It remains to invert this.

Let $\chi_{n}(t)$ be such that

$$
\int_{0}^{\infty} \chi_{n}(t) e^{-p t} d t=\frac{2}{\Omega a \gamma_{n}}\left\{\frac{e^{-\gamma_{n}|\nu|}}{p^{\frac{2}{2} \beta_{n}^{2}}+\alpha_{n}^{2}}+\frac{\gamma_{n} \sin \frac{p n \pi}{\Omega a}|y|}{\frac{p n \pi}{a \Omega}\left(p^{2} \beta_{n}^{2}+\alpha_{n}^{2}\right)}\right\} .
$$

Then

$$
\begin{aligned}
G= & \sum_{n=1}^{\infty}\left[\frac{\partial^{3}}{\partial x^{\prime}} \frac{\partial^{2}}{\partial t^{2}}+\Omega \frac{\partial^{2}}{\partial y \partial t}\right] \frac{n \pi}{\Omega a} \chi_{n}(t) \frac{\cos n \pi x}{a} \frac{\sin n \pi x^{\prime}}{a} \\
& +\sum_{n=1}^{\infty}\left[\frac{\partial}{\partial x^{\prime}} \frac{\partial}{\partial y} \frac{\partial}{\partial t}+\Omega \frac{\partial}{\partial y^{2}}\right] \chi_{n}(t) \frac{\sin n \pi x}{a} \frac{\sin n \pi x^{\prime}}{a} .
\end{aligned}
$$

Thus, if $\chi_{n}(t)$ is found, the problem is solved. 
The right-hand side of Eq. (34) may be written as

$$
\begin{aligned}
& =\frac{2}{\Omega a \sqrt{\frac{p^{2}}{c^{2}}+\alpha_{n}^{2}}}\left\{\frac{\exp -\left(p^{2} / c^{2}+\alpha_{n}^{2}\right)^{1 / 2}|y|}{p^{2} \beta_{n}^{2}+\alpha_{n}^{2}}+\frac{\left(p^{2} / c^{2}+\alpha_{n}^{2}\right)^{1 / 2} \sin \frac{p n \pi}{\Omega a}|y|}{\frac{p n \pi}{a \Omega}\left(p^{2} \beta_{n}^{2}+\alpha_{n}^{2}\right)}\right\} \\
& =\frac{2}{\left(p^{2}+\frac{\alpha_{n}^{2}}{\beta_{n}^{2}}\right)}\left\{\frac{c}{\Omega a \beta_{n}^{2}} \frac{\exp -\left(p^{2}+\alpha_{n}^{2} c^{2}\right)^{1 / 2}|y| / c}{\sqrt{p^{2}+\alpha_{n}^{2} c^{2}}}+\frac{1}{n \pi \beta_{n}^{2}} \frac{\sin \frac{p n \pi|y|}{\Omega a}}{p}\right\} .
\end{aligned}
$$

The inverse transform of $\left(p^{2}+\alpha_{n}^{2} / \beta_{n}^{2}\right)^{-1}$ is

$$
\frac{\beta_{n}}{\alpha_{n}} \sin \left(\frac{\alpha_{n}}{\beta_{n}} t\right)
$$

(see [4a]). The inverse transform of

$$
\frac{\exp -\left(p^{2}+\alpha_{n}^{2} c^{2}\right)^{1 / 2}|y| / c}{\left(p^{2}+\alpha_{n}^{2} c^{2}\right)^{1 / 2}}
$$

is

$$
J_{0}\left(\alpha_{n} c\left(t^{2}-|y|^{2} / c^{2}\right)^{1 / 2}\right) H\left(t-\frac{|y|}{c}\right)
$$

(see [4b]). The results (37a) and (37b) may be obtained readily from any good table of Laplace transforms. The inverse transform of

$$
\frac{\sin \frac{p n \pi|y|}{\Omega a}}{p}
$$

is

$$
\int_{0}^{\infty} J_{0}\left(2(s t)^{1 / 2}\right) \frac{d}{d s} \operatorname{bei}\left(\frac{2\left(s^{n \times|y|}\right)^{1 / 2}}{\Omega a}\right) d s .
$$

This does not seem to be a known result and the derivation is given in Appendix 1 .

It follows from the results $(37 \mathrm{a}, \mathrm{b}, \mathrm{c})$ and the Faltung theorem that the value of $\chi_{n}(t)$, being the inverse transform of the expression (36), is given by

$$
\begin{aligned}
\chi_{n}(t)= & \int_{0}^{t} \frac{\beta_{n}}{\alpha_{n}} \sin \frac{\alpha_{n}}{\beta_{n}}\left(t-t^{\prime}\right) \\
& \cdot\left\{\frac{c}{\Omega a \beta_{n}^{2}} J_{0}\left(\alpha_{n} c\left(t^{\prime 2}-|y|^{2} / c^{2}\right)^{1 / 2}\right) H\left(t^{\prime}-\frac{|y|}{c}\right)\right. \\
& \left.\left.+\frac{1}{n \pi \beta_{n}^{2}} \int^{\infty} J_{0}\left(2 s t^{\prime}\right)^{1 / 2}\right) \frac{d}{d s} b e i\left(2\left(\frac{s n \pi|y|}{\Omega a}\right)^{1 / 2}\right) d s\right\} d t^{\prime}
\end{aligned}
$$

Clearly $G\left(x, x^{\prime} ; y, y^{\prime} ; t\right)=G\left(x, x^{\prime} ; y-y^{\prime}, 0 ; t\right)$ as $y$ only occurs as $|y|$. A problem involving a flow across the side of the canal only, say, $x=0$, involves a simplification of $G$. 
In this case $x^{\prime}=0$ and

$$
\left[\frac{\partial}{\partial x^{\prime}}\left(\sin \frac{n \pi x^{\prime}}{a}\right)\right]_{x^{\prime}=0}=\left(\cos \frac{n \pi x^{\prime}}{a}\right)_{x^{\prime}=0} \frac{n \pi}{a}=\frac{n \pi}{a}
$$

and the expression (35) reduces to

$$
G(x, 0 ; y, 0 ; t)=\sum_{n=1}^{\infty} \frac{n^{2} \pi^{2}}{\Omega a} \chi_{n}^{\prime \prime}(t) \cos \frac{n \pi x}{a}+\sum_{n=1}^{\infty} \frac{n \pi}{a} \frac{\partial}{\partial y} \chi_{n}^{\prime}(t) \sin \frac{n \pi x}{a}
$$

4. Green's function for a semi-infinite ocean. Consider a semi-infinite ocean defined by $0<x$. Then the Green's function of interest is defined by

$$
\frac{\partial^{2} G}{\partial x^{2}}+\frac{\partial^{2} G}{\partial y^{2}}-K^{2} G-\frac{1}{c^{2}} \frac{\partial^{2} G}{\partial t^{2}}=\delta\left(x-x^{\prime}\right) \delta(y) \delta(t) \quad x, x^{\prime}>0
$$

with

$$
\frac{\partial^{2} G}{\partial x \partial t}-\Omega \frac{\partial G}{\partial y}=0 \quad \text { on } \quad x=0
$$

and

$$
G=0 \quad \partial G / \partial t=0 \quad t=0 .
$$

The transformed equations will be

$$
\frac{\partial^{2} \bar{G}}{\partial x^{2}}+\frac{\partial^{2} \bar{G}}{\partial y^{2}}-\gamma^{2} \bar{G}=\delta\left(x-x^{\prime}\right) \delta(y) \quad x, x>0
$$

and

$$
p \partial \bar{G} / \partial x-\Omega \partial \bar{G} / \partial y=0 \quad \text { on } \quad x=0
$$

where

$$
\gamma^{2}=K^{2}+p^{2} / c^{2}
$$

The solution of Eq. (41a) in an unbounded region is

$$
\begin{aligned}
\tilde{G}_{0} & =-\frac{1}{2 \pi} K_{0}\left(\gamma\left(\left(x-x^{\prime}\right)^{2}+y^{2}\right)^{1 / 2}\right) \\
& =-\frac{1}{4 \pi} \int_{-\infty}^{\infty} \frac{e^{i u \nu} \exp -\left(\left|x-x^{\prime}\right|\left(u^{2}+\gamma^{2}\right)^{1 / 2}\right) d u}{\left(\gamma^{2}+u^{2}\right)^{1 / 2}}
\end{aligned}
$$

(see [4c]). $G_{0}$ has been given in explicit form [5]. Let

$$
\bar{G}=\bar{G}_{0}+\bar{G}^{*}
$$

where $\bar{G}^{*}$ obeys

$$
\partial^{2} \bar{G}^{*} / \partial x^{2}+\partial^{2} \bar{G}^{*} / \partial y^{2}-\gamma^{2} \bar{G}^{*}=0 \text { in } x \geq 0 .
$$

$\bar{G}^{*}$ represents the disturbance reflected at $x=0$, so to speak, and will therefore die out as $x \rightarrow \infty$; and so

$$
\bar{G}^{*}=\int_{-\infty}^{\infty} e^{i u \nu} \exp -\left(x\left(u^{2}+\gamma^{2}\right)^{1 / 2}\right) g(u) d u
$$


It will be convenient (the reason for this will be seen later) to take $\bar{G}^{*}$ in the form

$$
\bar{G}^{*}=\left(p \frac{\partial}{\partial x}+\Omega \frac{\partial}{\partial y}\right)^{2} \int_{-\infty}^{\infty} \frac{e^{i u \nu} \exp -\left(\left(x+x^{\prime}\right)\left(u^{2}+\gamma^{2}\right)^{1 / 2}\right)}{4 \pi\left(u^{2}+\gamma^{2}\right)^{1 / 2}} \phi(u) d u .
$$

It is easily verified that the relation (43c) is equivalent to a special form of (43b).

From the condition (41b), it follows that

$$
p \frac{\partial \bar{G}}{\partial x}-\Omega \frac{\partial \bar{G}}{\partial y}=\frac{1}{4 \pi} \int_{-\infty}^{\infty} \frac{e^{i u \nu} \exp -x^{\prime}\left(u^{2}+\gamma^{2}\right)^{1 / 2}}{\left(u^{2}+\gamma^{2}\right)^{1 / 2}} \chi(u) d u
$$

where

$$
\begin{aligned}
\chi(u)=(-p & \left.\left(u^{2}+\gamma^{2}\right)^{1 / 2}+i u \Omega\right) \exp x\left(u^{2}+\gamma^{2}\right)^{1 / 2} \\
& +\left(-p\left(u^{2}+\gamma^{2}\right)^{1 / 2}-i u \Omega\right)\left(p\left(u^{2}+\gamma^{2}\right)^{1 / 2}-i u \Omega\right)^{2} \exp -x\left(u^{2}+\gamma^{2}\right)^{1 / 2} \phi(u) .
\end{aligned}
$$

Now on $x=0$, it follows that $\chi(u)$ must vanish and so

$$
\phi(u)=-\frac{1}{p^{2}\left(u^{2}+\gamma^{2}\right)+u^{2} \Omega^{2}} .
$$

The reason for the form (43c) is now seen. It follows that

$G^{*}=-\left(p \frac{\partial}{\partial x}+\Omega \frac{\partial}{\partial y}\right)^{2} \frac{1}{4 \pi} \int_{-\infty}^{\infty} \frac{e^{i u \nu} \exp -\left(x+x^{\prime}\right)\left(u^{2}+\gamma^{2}\right)^{1 / 2}}{\left(u^{2}+\gamma^{2}\right)^{1 / 2}}-\frac{1}{p^{2}\left(u^{2}+\gamma^{2}\right)+u^{2} \Omega^{2}} d u$.

Let

$$
\frac{1}{2 \pi} \int_{-\infty}^{\infty} \frac{e^{i u \eta}}{p^{2}\left(u^{2}+\gamma^{2}\right)+u^{2} \Omega^{2}} d u=g(\eta) .
$$

Then, by the Faltung theorem [6]

$$
\bar{G}^{*}=-\left(p \frac{\partial}{\partial x}+\Omega \frac{\partial}{\partial y}\right)^{2} \frac{1}{4 \pi} \cdot 2 \pi \int_{-\infty}^{\infty} \frac{1}{\pi} K_{0}\left(\gamma\left(\left(x+x^{\prime}\right)^{2}+(y-\eta)^{2}\right)\right)^{1 / 2} g(\eta) d \eta .
$$

Also,

$$
g(\eta)=\frac{1}{2\left(p^{2}+\Omega^{2}\right)^{1 / 2} p \gamma} \exp \left\{\frac{-p \gamma|\eta|}{\left(p^{2}+\Omega^{2}\right)^{1 / 2}}\right\}
$$

(see $[4 \mathrm{~d}]$ ), and so

$$
\begin{aligned}
\bar{G}^{*}=-\left(p \frac{\partial}{\partial x}+\Omega\right. & \left.\frac{\partial}{\partial y}\right)^{2} \\
& \cdot \frac{1}{2} \int_{-\infty}^{\infty} \frac{K_{0}\left(\gamma\left(\left(x+x^{\prime}\right)^{2}+(y-\eta)^{2}\right)^{1 / 2}\right)}{\left(p^{2}+\Omega^{2}\right)^{1 / 2} p \gamma} \exp \left\{\frac{-p \gamma|\eta|}{\left(p^{2}+\Omega^{2}\right)^{1 / 2}}\right\} d \eta
\end{aligned}
$$

where

$$
\gamma^{2}=K^{2}+\frac{p^{2}}{c^{2}}=\frac{1}{c^{2}}\left(\Omega^{2}+p^{2}\right)
$$

One thing comes out of this analysis immediately, namely that the image system in the region $x<0$ which makes the satisfaction of the boundary condition at $x=0$ possible 
consists of a row of sources which are situated along the line $x=-x^{\prime}$, and not of a single image source at the point $\left(-x^{\prime}, 0\right)$.

The inversion proceeds as follows:

$$
\begin{aligned}
\bar{G}^{*}=-\left(p \frac{\partial}{\partial x}+\Omega\right. & \left.\frac{\partial}{\partial y}\right)^{2} \frac{c}{2 p\left(p^{2}+\Omega^{2}\right)} \\
& \cdot \int_{-\infty}^{\infty} K_{0}\left(\left(p^{2}+\Omega^{2}\right)^{1 / 2} c^{-1}\left(\left(x+x^{\prime}\right)^{2}+(y-\eta)^{2}\right)^{1 / 2}\right) e^{-p(|\eta| / c)} d \eta
\end{aligned}
$$

If $F\left(x, x^{\prime}, y, t\right)$ is the inverse transform of the infinite integral, that of

$$
\frac{\Omega}{p^{2}+\Omega^{2}} \int_{-\infty}^{\infty} K_{0}\left(\left(p^{2}+\Omega^{2}\right)^{1 / 2} c^{-1}\left(\left(x+x^{\prime}\right)^{2}+(y-\eta)^{2}\right)^{1 / 2}\right) e^{-p(1 / 1 / c)} d \eta
$$

is

$$
A\left(x, x^{\prime}, y, t\right)=\int_{0}^{t} \sin \Omega\left(t-t^{\prime}\right) F\left(x, x^{\prime}, y, t^{\prime}\right) d t^{\prime}
$$

This follows immediately by the Faltung theorem for the inversion of Laplace transforms. Clearly

$$
A\left(x, x^{\prime}, y, 0\right)=0
$$

Inverting the operator

$$
-\frac{c}{2}\left(p \frac{\partial^{2}}{\partial x^{2}}+2 \Omega \frac{\partial^{2}}{\partial x \partial y}+\frac{\Omega^{2}}{p} \frac{\partial^{2}}{\partial y^{2}}\right)
$$

it follows, using Eq. (49), that

$$
G^{*}\left(x, x^{\prime}, y, t\right)=-\frac{c}{2 \Omega}\left[\frac{\partial^{3} A}{\partial x^{2} \partial t}+2 \Omega \frac{\partial^{2} A}{\partial x \partial y}+\Omega^{2} \int_{0}^{t} \frac{\partial^{2} A}{\partial y^{2}}\left(x, x^{\prime}, y, \tau\right) d \tau\right]
$$

It remains to determine

$$
\begin{aligned}
F\left(x, x^{\prime}, y, t\right) & =\int_{-\infty}^{\infty} K_{0}\left(\left(p^{2}+\Omega^{2}\right)^{1 / 2} c^{-1}\left(\left(x+x^{\prime}\right)^{2}+(y-\eta)^{2}\right)^{1 / 2}\right) e^{-p(|\eta| / c)} d \eta \\
& =\int_{-\infty}^{\infty} K_{0}\left(\left(p^{2}+\Omega^{2}\right)^{1 / 2} b\right) e^{-p(|\eta| / c)} d \eta, \text { say. }
\end{aligned}
$$

The inverse Laplace transform of $K_{0}\left(\left(p^{2}+\Omega^{2}\right)^{1 / 2} b\right)$ can be seen to be [4e]

$$
\frac{\cos \left(\Omega\left(t^{2}-b^{2}\right)^{1 / 2}\right)}{\left(t^{2}-b^{2}\right)^{1 / 2}} t>b, \quad 0<t<b .
$$

It follows therefore that

$$
F\left(x, x^{\prime}, y, t\right)=\int \frac{\cos \left(\Omega\left(\left(t^{2}-|\eta|^{2} / c\right)-b^{2}\right)^{1 / 2}\right.}{\left((t-|\eta| / c)^{2}-b^{2}\right)^{1 / 2}} d \eta
$$

where the integral is taken over the values of $\eta$ defined by

$$
t-\frac{|\eta|}{c}>\frac{1}{c}\left\{\left(x+x^{\prime}\right)^{2}+(y-\eta)^{2}\right\}^{1 / 2} \text {. }
$$


Appendix. The inverse transform of

$$
p^{-1} \sin \left(\alpha p^{-1}\right) \quad \text { is } \quad b e i\left(2(\alpha t)^{1 / 2}\right)
$$

[6] and hence that of

$$
\sin \left(\alpha p^{-1}\right) \quad \text { is } \quad \frac{d}{d t} b e i\left(2(\alpha t)^{1 / 2}\right)
$$

as

$$
\left[\frac{d}{d t} b e i\left(2(\alpha t)^{1 / 2}\right)\right]_{t=0}
$$

vanishes. Now if $f(t)$ is the inverse transform of $g(p)$, the inverse transform of $(1 / p) g\left(p^{-1}\right)$ is

$$
\int_{0}^{\infty} J_{0}\left(2(s t)^{1 / 2}\right) f(s) d s
$$

(see [4f]) and so the inverse transform of $p^{-1} \sin \alpha p$ is

$$
\int_{0}^{\infty} J_{0}\left(2(s t)^{1 / 2}\right) \frac{d}{d s} b e i\left(2(\alpha s)^{1 / 2}\right) d s .
$$

\section{References}

[1] J. Proudman, Dynamical oceanography, Methuen, London, 1953, p. 220.

[2] Ll. G. Chambers, J. Fluid Mech. 22, 209 (1965)

[3] L. Ainola, Eesti NSV Tead. Akad. Toimetised Füüs-Mat. Tehn.-Tead. Seer. 16, 139 (1967)

[4] W. Magnus, and F. Oberhettinger, Formeln and Sätze für die speziellen Funktionen der mathematischen Physik, Springer-Verlag, Berlin,1948; a, p. 171 ; b, p. 179 ; c, p. 164 ; d, p. 162 ; e, p. 179 ; f, p. 169

[5] Ll. G. Chambers, Proc. Edinburgh Math. Soc. 15, 125 (1966)

[6] J. Irving, and N. Mullineux, Mathematics in physics and engineering, Academic Press, New York, 1959 , p. 593

[7] Bateman Manuscript Project, Tables of Integral Transforms, I, McGraw-Hill, New York, 1954, p. 253 